\title{
MODELAGEM, CONSTRUÇÃO E ANÁLISE DE DESEMPENHO DO FLICKERMETER IEC
}

\author{
José Rubens Macedo Jr.* \\ jrubens.macedo@gmail.com
}

Gilberto C. Drumond Sousa ${ }^{\dagger}$

g.sousadele.ufes.br

Emerson Trarbach ${ }^{\dagger}$

emerson.trarbach@gmail.com

\author{
Guilherme P. Colnago ${ }^{\dagger}$ \\ guilhermecolnago@gmail.com \\ José Luiz de Freitas Vieira ${ }^{\dagger}$ \\ joseluiz@ele.ufes.br \\ Domingos S. L. Simonetti ${ }^{\dagger}$ \\ d.simonetti@ele.ufes.br
}

*Faculdade de Engenharia Elétrica, UFU, Av. João Naves de Ávila, 2121, Campus Santa Mônica, Uberlândia - MG

${ }^{\dagger}$ Departamento de Engenharia Elétrica, UFES, Av. Fernando Ferrari, s/n, Campus Universitário, Vitória - ES

\section{RESUMO}

O presente trabalho apresenta a modelagem passo a passo do flickermeter baseado no protocolo IEC 61000-4-15, implementado em ambiente computacional Matlab-Simulink ${ }^{\circledR}$. O referido modelo constitui uma ferramenta de grande utilidade nas simulações e estudos associados com a qualidade da energia elétrica, notadamente relacionados com o fenômeno da flutuação de tensão nos sistemas de potência. Adicionalmente, o trabalho apresenta uma nova proposta de implementação do bloco 5 do flickermeter IEC, responsável pela análise estatística dos registros de sensação instantânea de flicker e pelo cálculo do indicador de severidade de flicker de curta duração (Pst). Por fim, são apresentados os resultados dos testes de calibração do modelo desenvolvido, assim como os testes de desempenho de um medidor (hardware) desenvolvido utilizando-se o modelo de flickermeter proposto no presente trabalho.

PALAVRAS-CHAVE: Flickermeter, flutuação de tensão, flicker, Matlab-Simulink.

Artigo submetido em 29/10/2009 (Id.: 01071)

Revisado em 02/03/2010, 20/08/2010

Aceito sob recomendação do Editor Associado Prof. Enes Gonçalves Marra

\section{ABSTRACT}

IEC flickermeter modeling, construction and test of performance

This work deals with a step-by-step modeling and implementation of IEC flickermeter. The developed Matlab-Simulink flickermeter model could be considered an important tool to several power quality analyses related to voltage fluctuations. In addition, this work proposes a different way to implementation of flickermeter's block 5, which one is responsible for the computation of probability short term flicker index (Pst). Finally, calibration tests results and also the behavior analisys of an implemented flickermeter device, considering the proposed modeling, are discussed.

KEYWORDS: Flickermeter, voltage fluctuation, flicker, Matlab-Simulink.

\section{INTRODUÇÃO}

Os estudos e simulações computacionais relacionados com a qualidade da energia elétrica são complexos e exigem modelos e sistemas computacionais muito específicos. Comumente, os estudos associados com o fluxo de frequências harmônicas nas redes elétricas, por exemplo, são substanciados por uma vasta quantidade de softwares 
e sistemas computacionais amplamente disponíveis aos engenheiros e pesquisadores do assunto. Para a questão do desequilíbrio de tensão, assim como das variações de tensão de curta duração, os mesmos estudos podem ser realizados através de usos diferenciados dos tradicionais modelos de fluxo de carga e curto-circuito, também amplamente disponíveis. No entanto, para o caso específico das flutuações de tensão, notadamente em função de sua modelagem matemática e implementação relativamente complexa, poucos são os aplicativos disponíveis. Assim, o desenvolvimento computacional do protocolo definido pelo padrão IEC 61.000-4-15 tem por objetivo disponibilizar uma ferramenta de grande utilidade aos estudos relacionados com o fenômeno das flutuações de tensão nas redes elétricas. A escolha pelo ambiente de programação Matlab-Simulink ${ }^{\circledR}$ é consubstanciada pela grande aceitação da ferramenta nos estudos dos sistemas elétricos de potência (Freitas e França, 2005; Bhim et al. 2006; Patel e Pagalthivarthi, 2005).

\section{O PROTOCOLO IEC 61.000-4-15}

A necessidade de se ter uma padronização internacional para quantificação das flutuações de tensão tornou-se evidente quando do aparecimento dos primeiros compensadores estáticos tiristorizados como, o reator controlado a tiristores (RCT) e os capacitores chaveados por tiristores (CCT) (Deckmann, 1999). Assim, em 1986 foi publicado o documento "IEC Publication 868 - Flickermeter - Functional and design especifications" (IEC, 1986), atualmente revisado como International Standard IEC 61000-4-15 "Testing and measurement techniques - Section 15: Flickermeter - Functional and design specifications" (IEC, 2003). A quantificação das flutuações de tensão é atualmente realizada por meio deste protocolo IEC em vários países do mundo, incluindo-se o Brasil.

O diagrama funcional do fickermeter definido pelo protocolo IEC 61000-4-15 pode ser simplificado de forma a tornar seu entendimento mais simplificado, conforme diagrama de blocos mostrado na figura 1 .

\section{A 1 B 2 C 3 D 4 E 5 F BLOCOS \\ 1. Adaptação da tensão de entrada 2. Demodulação do sinal \\ 3. Ponderação em frequência \\ 4. Média quadrática \\ 5. Tratamento estatístico \\ ENTRADA/SAÍDA DOS BLOCOS \\ A. Tensão de entrada \\ B. Tensão de entrada normalizada \\ C. Flutuação de tensão \\ D. Flutuação de tensão ponderada \\ E. Sensação instantânea de flicker \\ F. Severidade de flicker (Pst)}

Figura 1: Diagrama simplificado do flickermeter IEC.
Conforme mostrado na figura 1, o protocolo do flickermeter é composto por cinco blocos estruturais, os quais compõem o modelo efetivamente, assim como seis saídas específicas, cujos resultados possuem significados físico-matemáticos distintos. Em linhas gerais, pode-se também dividir o protocolo do fickermeter da IEC em duas partes distintas, a saber:

- Simulação da resposta do sistema olho-lâmpada-cérebro para o fenômeno da cintilação luminosa;

- Análise estatística em tempo real da cintilação luminosa e apresentação dos resultados.

A primeira parte é composta pelo conjunto de blocos 2, 3 e 4 do diagrama simplificado indicado na figura 1 . O bloco 5 , por sua vez, compõe a segunda parte do protocolo doflickermeter IEC.

Uma observação importante está relacionada com as saídas $E$ e $F$ indicadas na figura 1, as quais representam a sensação instantânea de flicker (Sf) e a severidade de flicker (Pst), respectivamente. A sensação instantânea de flicker está relacionada com a percepção visual, para o observador humano, da modulação do fluxo luminoso em decorrência da modulação do valor eficaz (ou de pico) da tensão. Um valor unitário para a sensação instantânea de flicker representa o limite de perceptividade para 50\% dos observadores em eventuais condições de teste.

A severidade de flicker (saída $F$ ), por sua vez, representa um valor matemático baseado no comportamento estatístico da sensação instantânea de flicker (saída $E$ ). Os limites atribuídos para os indicadores de severidade de flicker (Pst e Plt), baseiam-se no comportamento estatístico da sensação instantânea de flicker $(S f)$. Onde o Pst é o indicador de severidade de curta duração e o Plt é o indicador de severidade de longa duração. Na seção seguinte serão detalhados os processos construtivos, em ambiente Matlab-Simulink ${ }^{\circledR}$, dos cinco blocos funcionais que constituem o referido protocolo.

\section{DESENVOLVIMENTO COMPUTACIONAL DO FLICKERMETER CONFORME O PROTOCOLO IEC 61.000-4-15}

A figura 2 ilustra o esquema de blocos do flickermeter desenvolvido. Em um primeiro momento será detalhada apenas a implementação dos blocos 1 a 4 e, posteriormente, devido ao seu caráter diferenciado, serão abordadas as questões associadas à implementação do bloco 5 . Em 
termos práticos, os blocos 1, 2, 3 e 4 foram implementados em ambiente simulink, utilizando-se modelos e funções já disponíveis no referido aplicativo e o bloco 5, por sua vez, foi implementado através de linhas de código na área de trabalho do matlab.

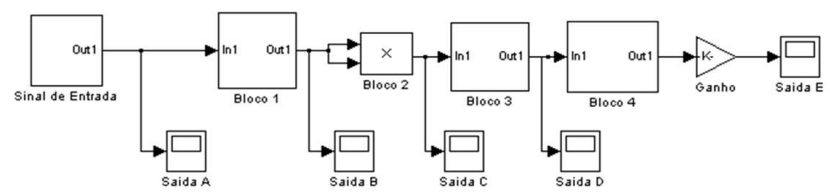

Figura 2: Diagrama de blocos do flickermeter desenvolvido.

Para efeito dos testes de calibração do modelo, o sinal de entrada aplicado ao bloco 1 representa a tensão da rede, em 127 Volts (fase-neutro), sobreposta por um sinal de modulação, conforme mostrado na figura 3 .

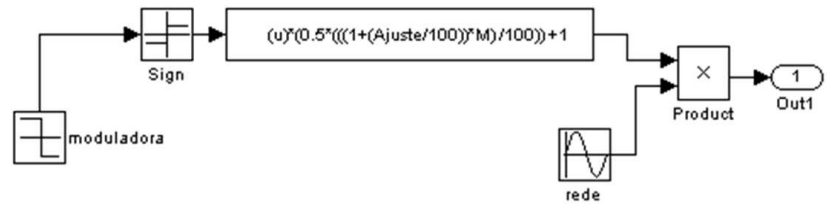

Figura 3: Diagrama de blocos do sinal de entrada com modulação quadrática.

As curvas indicadas na figura 4 ilustram uma condição particular da tensão de entrada, modulada com uma amplitude $\Delta \mathrm{V} / \mathrm{V}$ igual a $0,253 \%$, na frequência de 8,8 $\mathrm{Hz}$, a qual segundo o protocolo IEC deverá resultar uma sensação instantânea de flicker (saída $E$ ) unitária (1,0pu). Essa condição particular da tensão de entrada será utilizada nas próximas etapas da modelagem para demonstração do flickermeter desenvolvido.

\subsection{Implementação do bloco (adaptação da tensão de entrada)}

O bloco 1 do modelo do flickermeter tem por objetivo normalizar a tensão de entrada $v(t)$ por um sinal de referência Vref. A figura 5 ilustra o diagrama de blocos desenvolvido no simulink ${ }^{\circledR}$ para o adaptador da tensão de entrada.

Considerando-se a existência de diferentes níveis padronizados para o fornecimento de energia elétrica
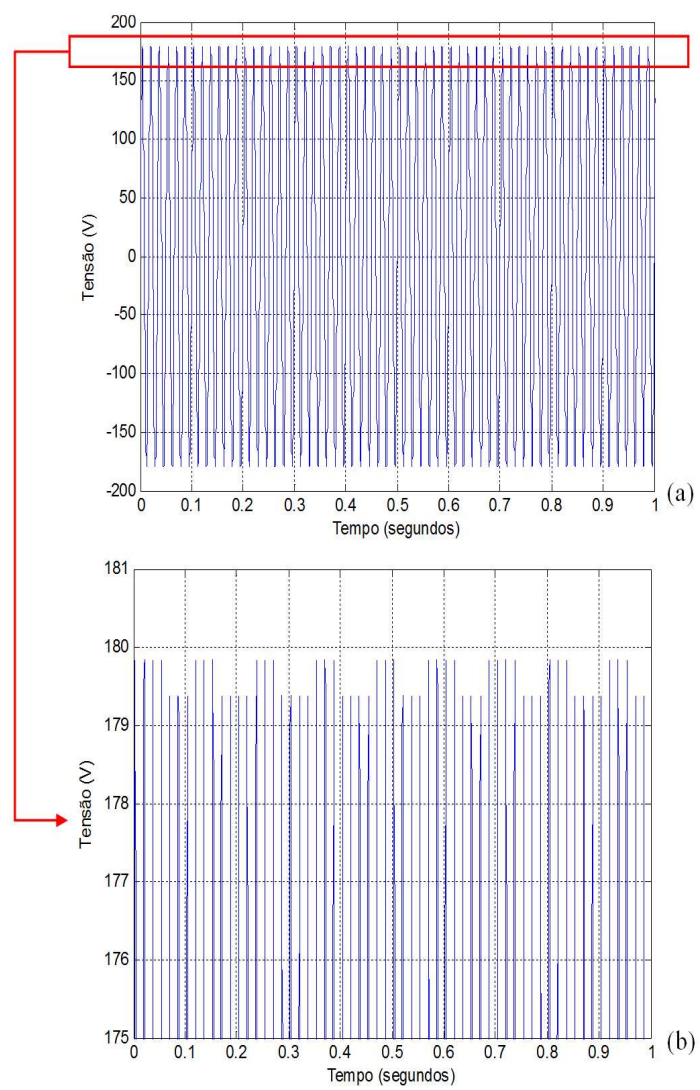

Figura 4: Exemplo de modulação retangular da tensão de entrada (Saída $A$ ). (a) forma de onda da tensão, (b) alteração da escala para melhor visualização da modulação retangular.

em baixa tensão, existe a necessidade de normalizar as variações de tensão e expressá-las em termos percentuais de uma tensão de referência (Vref). Isso permite, entre outras vantagens, comparar diretamente os efeitos das mesmas variações relativas, observadas em diferentes níveis de tensão.

A adaptação da tensão de entrada indicada no protocolo da IEC consiste na normalização da tensão medida pelo valor obtido através de um processador de média móvel com tempo de acomodação de 1 minuto. Em termos práticos, isto significa que variações lentas, com tempo de acomodação

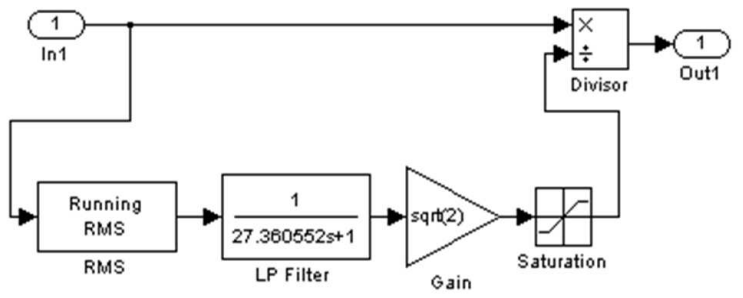

Figura 5: Diagrama de blocos do adaptador da tensão de entrada. 
maior que 1 minuto, não contribuem para o cálculo da severidade de flicker (Deckmann, 1999). Variações mais rápidas, por sua vez, são expressas em termos percentuais da tensão da referência móvel, conforme indicado em (1).

$$
\Delta V \%=\frac{V-\text { Vref }}{\text { Vref }} \times 100
$$

Onde:

$V=$ tensão fornecida pela rede local;

Vref = tensão de referência do último minuto (referência móvel);

A partir do sinal de entrada, o sinal de referência é calculado considerando-se o valor eficaz da tensão em análise, seguindo-se um filtro passa-baixas o qual, conforme especificado pelo protocolo IEC 61.000-4-15, representa um sistema de tempo de resposta de $10 \%$ a $90 \%$ do valor final igual a um minuto, para uma variação em degrau da tensão eficaz do sinal de entrada. A função de transferência para o filtro especificado é indicada em (2).

$$
F(s)=\frac{1}{27,360552 . s+1}
$$

A figura 6 ilustra a resposta em frequência para o referido filtro passa-baixas com freqüência de corte igual a 0,005830 Hz. Após o bloco 1 (saída $B$ ), tem-se uma tensão normalizada em função da tensão de referência. A figura 7 ilustra o sinal de saída do bloco 1 para uma tensão de entrada com modulação retangular, conforme mostrado na figura 4(b).

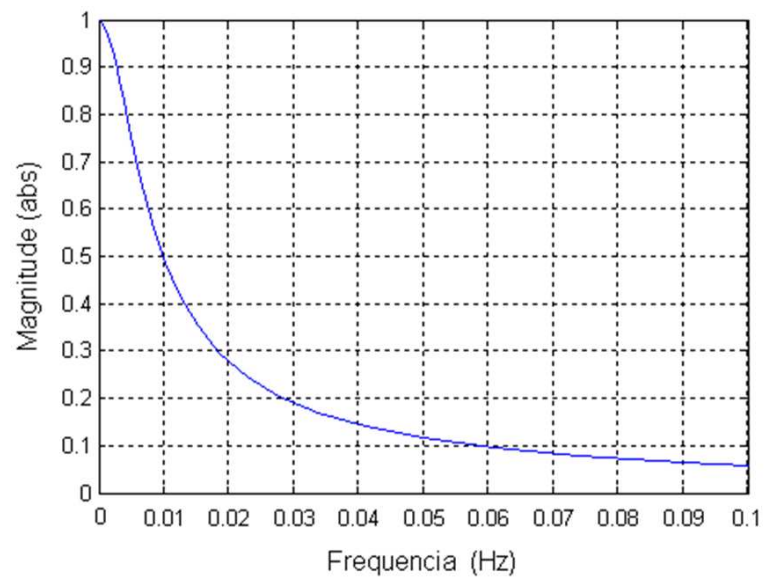

Figura 6: Resposta em frequência do filtro passa-baixas do bloco 1.

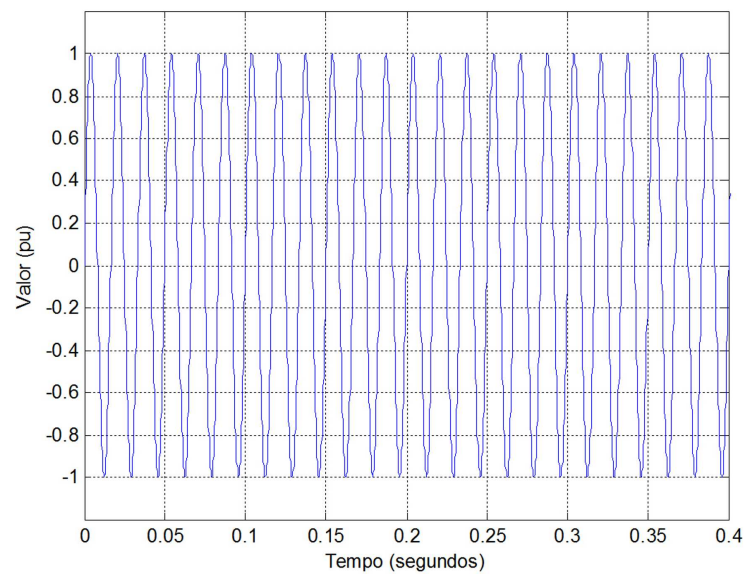

Figura 7: Tensão normalizada na saída do bloco 1 (Saída B).

\subsection{Implementação do Bloco (Demodulador quadrático)}

A função do bloco 2 é extrair a flutuação de tensão existente no sinal de tensão normalizado pela referência móvel. Nesse sentido, considerando-se a tensão modulante indicada na equação (3), tem-se que o objetivo do bloco 2 é simplesmente extrair a amplitude de modulação. Para esse propósito podem ser utilizados diferentes métodos (Bollen e Gu, 2006), sendo a maioria deles muito comuns nos sistemas de telecomunicação. Entretanto, a forma mais prática e simplificada para extração da modulação $m$, também utilizada no protocolo $I E C$, é a demodulação quadrática, a qual consiste simplesmente no quadramento do sinal de tensão normalizado pela referência móvel.

$$
v(t)=A \cdot\left(\cos \omega_{p} \cdot t\right) \cdot\left(1+m \cdot \cos \omega_{m} \cdot t\right)
$$

Onde:

$A=$ constante;

$v(t)=$ tensão instantânea de suprimento;

$\omega_{p}=$ frequência angular da tensão de suprimento (portadora);

$m$ = amplitude de modulação;

$\omega_{m}=$ frequência angular de modulação.

A forma utilizada para implementação do bloco 2 do fickermeter IEC no Simulink ${ }^{\circledR}$ é indicada na figura 8.

A curva mostrada na figura 9 indica o resultado de saída do bloco 2, considerando-se a tensão de entrada indicada na figura 4. 


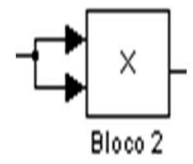

Figura 8: Diagrama de blocos do demodulador quadrático (bloco 2).

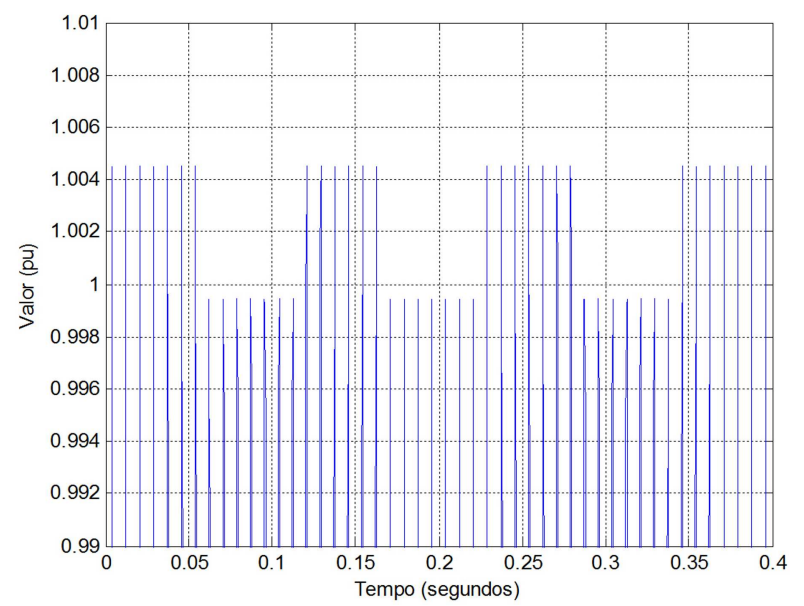

Figura 9: Tensão na saída do bloco 2 do flickermeter (Saída C).

\subsection{Implementação do Bloco 3 (Ponderação em frequência)}

O bloco 3 do flickermeter IEC representa o principal componente na modelagem do conjunto olho-lâmpada-cérebro. O objetivo deste bloco é proporcionar uma atenuação de 90 dB no sinal de entrada (IEC, 2003), a qual é produzida por um filtro específico de ponderação em frequência. Além do filtro de ponderação em frequência, contudo, outros dois filtros compõem a estrutura funcional do bloco.

Assim, o bloco 3 do flickermeter IEC é composto pelos seguintes filtros:

- Filtro passa-altas, com frequência de corte igual a 0,05 $\mathrm{Hz}$;

- Filtro passa-baixas, tipo Butterworth de $6^{a}$ ordem, com frequência de corte igual a $42 \mathrm{~Hz}$ (para redes de $120 \mathrm{~V} / 60 \mathrm{~Hz}$ ) ou $35 \mathrm{~Hz}$ (para redes de 230V/50 Hz);

- Filtro de ponderação em frequência.

A estrutura funcional para o bloco 3 do modelo do flickermeter IEC, composto pelos três filtros indicados acima, é mostrada na figura 10.

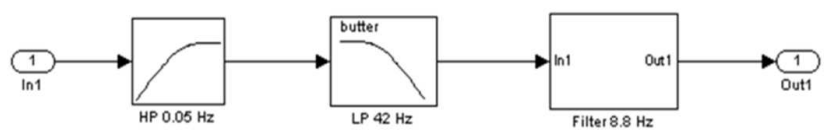

Figura 10: Diagrama de blocos para ponderação em frequência (bloco 3).

A função de transferência para o filtro passa-altas, com frequência de corte igual a $0,05 \mathrm{~Hz}$, é indicada em (4). A figura 11, por sua vez, ilustra a reposta em frequência obtida para o referido filtro.

$$
F(s)=\frac{3,175 . s}{3,175 . s+1}
$$

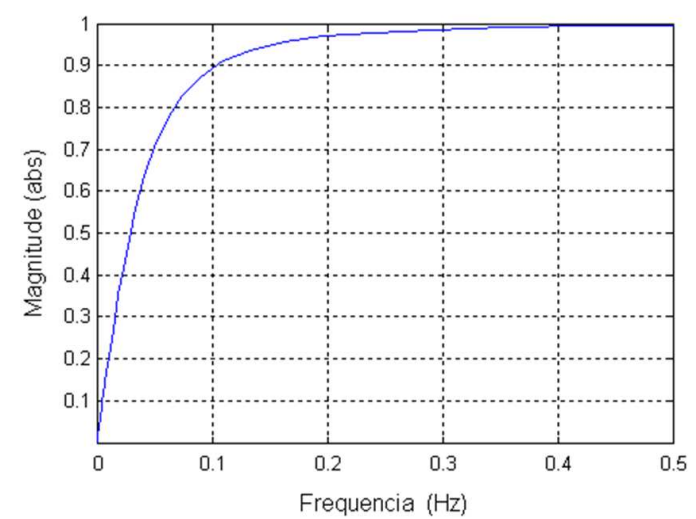

Figura 11: Resposta em frequência do filtro passa-altas do bloco 3.

Para o projeto do filtro passa-baixas, representado por um filtro Butterworth de $6^{a}$ ordem, a respectiva função de transferência pode ser obtida, em termos matemáticos, a partir da série de polinôminos indicados a seguir (Bertola et al., 2004).

$$
F(s)=\prod_{i=1}^{3}\left[\frac{\omega_{i}^{2}}{\left(\frac{s}{\omega_{C}}\right)^{2}+2 \cdot \beta_{i} \cdot \omega_{i} \cdot\left(\frac{s}{\omega_{C}}\right)+\omega_{i}^{2}}\right]
$$

Onde:

$s=$ variável complexa de Laplace;

$\omega_{C}=$ freqüência de corte $(\mathrm{em} \mathrm{rad} / \mathrm{seg})$;

$\omega_{1}=\omega_{2}=\omega_{3}=1$; 
$\beta_{1}=0,26$;

$\beta_{2}=0,71 ;$

$\beta_{3}=0,97$

Os parâmetros $\beta_{1}, \beta_{2}$ e $\beta_{3}$ são definidos segundo (Bertola et al., 2004). Assim, para um filtro Butterworth de $6^{a}$ ordem, e para uma frequência de corte igual a $42 \mathrm{~Hz}$ (ou 2. $\pi .42 \mathrm{rad} / \mathrm{seg}$ ), utilizada para o caso de modelagem considerando-se lâmpadas de $120 \mathrm{~V} \mathrm{/} \mathrm{60Hz,} \mathrm{tem-se} \mathrm{a}$ seguinte função de transferência após a substituição dos valores correspondentes em (5):

$$
F 42(H z)=\frac{1}{F A} \cdot \frac{1}{F B} \cdot \frac{1}{F C}
$$

Onde:

$$
\begin{aligned}
& F A=\frac{1}{\left(0,0000143596 . s^{2}+0,00197049 . s+1\right)} \\
& F B=\frac{1}{\left(0,0000143596 . s^{2}+0,00538095 . s+1\right)} \\
& F C=\frac{1}{\left(0,0000143596 . s^{2}+0,00735144 . s+1\right)}
\end{aligned}
$$

Para o caso de uma rede elétrica considerando-se a modelagem da lâmpada em $230 \mathrm{~V} / 50 \mathrm{~Hz}$, a frequência de corte do filtro passa-baixas será de $35 \mathrm{~Hz}$ (ou 2. $\pi .35 \mathrm{rad} / \mathrm{seg}$ ), o que resulta na nova função de transferência indicada a seguir.

$$
F 35(H z)=\frac{1}{F A^{\prime}} \cdot \frac{1}{F B^{\prime}} \cdot \frac{1}{F C^{\prime}}
$$

Onde:

$$
\begin{aligned}
& F A^{\prime}=\frac{1}{\left(0,0000206778 . s^{2}+0,00236588 . s+1\right)} \\
& F B^{\prime}=\frac{1}{\left(0,0000206778 . s^{2}+0,00645714 . s+1\right)} \\
& F C^{\prime}=\frac{1}{\left(0,0000206778 . s^{2}+0,00882173 . s+1\right)}
\end{aligned}
$$

Para os propósitos do presente trabalho, cujo objetivo é a implementação do protocolo do flickermeter IEC em ambiente Simulink-Matlab ${ }^{\circledR}$, todos os filtros serão projetados para a redes de $120 \mathrm{~V} / 60 \mathrm{~Hz}$. Assim, no presente caso, portanto, o filtro passa-baixas tipo Butterworth de $6^{a}$ ordem a ser utilizado possui frequência de corte igual a 42 Hz. A figura 12 mostra a resposta em frequência do filtro, para as duas frequências de corte possíveis.

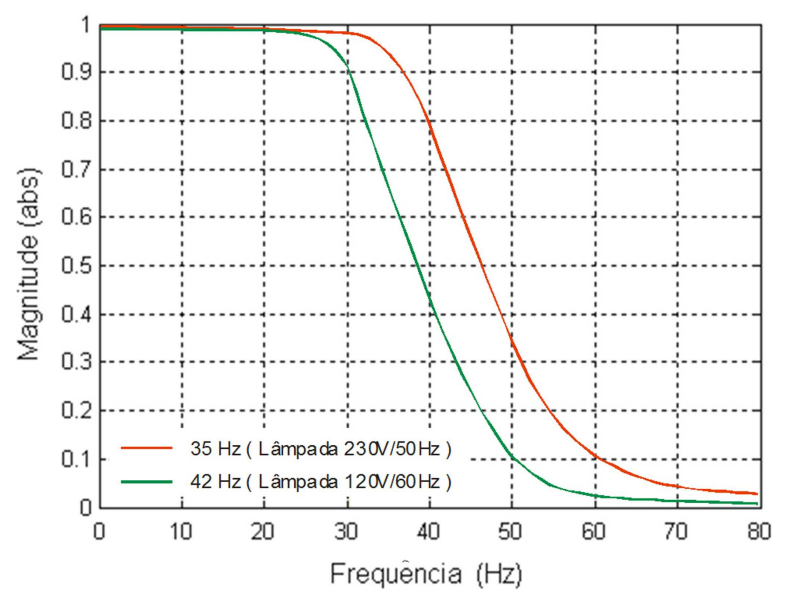

Figura 12: Resposta em freqüência do filtro Butterworth passa-baixas, com frequências de corte iguais a $35 \mathrm{~Hz}$ $(230 \mathrm{~V} / 50 \mathrm{~Hz})$ e $42 \mathrm{~Hz}(120 \mathrm{~V} / 60 \mathrm{~Hz})$.

Para uma melhor ilustração da função desses filtros, considera-se o sinal de entrada do bloco 3 , o qual é o resultado da quadratura do sinal indicado em (3), conforme a seguir:

$$
\begin{aligned}
v^{2}(t)=[ & \left.A^{2} \cdot \cos ^{2}\left(\omega_{p} \cdot t\right)\right] \times \\
& {\left[1+2 \cdot m \cdot \cos \left(\omega_{m} t\right)+m^{2} \cdot \cos ^{2}\left(\omega_{m} t\right)\right] }
\end{aligned}
$$

Ou ainda:

$$
\begin{aligned}
v^{2}(t)= & \frac{A^{2}}{2} \cdot\left[1+\cos \left(2 \cdot \omega_{p} \cdot t\right)\right] \times \\
& {\left[1+2 \cdot m \cdot \cos \left(\omega_{m} \cdot t\right)+m^{2} \cdot \cos ^{2}\left(\omega_{m} \cdot t\right)\right] }
\end{aligned}
$$

Da equação (15) podem ser identificados os seguintes termos:

- $\frac{A^{2}}{2}$, representando o nível CC;

- $\frac{A^{2}}{2} \cdot \cos \left(2 \cdot \omega_{p} \cdot t\right)$,representando um termo de dupla frequência; 
E, finalmente:

- $2 . m \cdot \frac{A^{2}}{2} \cdot \cos \left(\omega_{m} \cdot t\right), \quad$ representando o termo modulante.

Vale ainda observar que os termos remanescentes tornam-se desprezíveis para níveis de modulação muito baixos.

Com base nestas constatações, verifica-se que a função do filtro passa-altas, com frequência de corte igual a $0,05 \mathrm{~Hz}$ é simplesmente eliminar o nível CC. Adicionalmente, o filtro passa-baixas, tipo Butterworth de $6^{a}$ ordem, tem por objetivo eliminar o termo de dupla frequência da portadora (tensão de suprimento).

Assim, eliminando-se a componente de corrente contínua e o termo de dupla frequência da portadora, resulta:

$$
v(t)=m \cdot A^{2} \cdot \cos \left(\omega_{m} \cdot t\right)
$$

Normalizando o sinal indicado em (16) pela amplitude do sinal da portadora (tensão de suprimento), resulta:

$$
v(t)=m \cdot A \cdot \cos \left(\omega_{m} \cdot t\right)
$$

A equação (17) representa, portanto, o sinal modulante normalizado. Finalmente, a última etapa do bloco 3 do flickermeter IEC é representada pelo filtro de ponderação em frequência, o qual representa a resposta do sistema olho-lâmpada-cérebro em relação às variações de intensidade luminosa em lâmpadas incandescentes. O referido filtro é constituído por uma curva de ponderação em freqüência, cuja função de transferência é definida pelo próprio protocolo IEC 61.000-4-15. A estrutura da função de transferência para esse filtro é a seguinte:

$$
F(s)=\frac{k \cdot \omega_{1} \cdot s}{s^{2}+2 \cdot \lambda \cdot s+\omega_{1}^{2}} \cdot \frac{1+\frac{s}{\omega_{2}}}{\left(1+\frac{s}{\omega_{3}}\right) \cdot\left(1+\frac{s}{\omega_{4}}\right)}
$$

Onde os parâmetros associados, dependendo do tipo de lâmpada de referência a ser utilizada, são estabelecidos pelo protocolo IEC 61.000-4-15 (IEC, 2003), conforme indicado na tabela 1.

Tabela 1: Variáveis definidas pela IEC para o filtro de ponderação em frequência, dependendo do tipo de lâmpada.

\begin{tabular}{|c|c|c|}
\hline Variável & Lâmpada $230 \mathrm{~V} / 50 \mathrm{~Hz}$ & Lâmpada $120 \mathrm{~V} / 60 \mathrm{~Hz}$ \\
\hline$k$ & 1,74802 & 1,6357 \\
\hline$\lambda$ & $2 . \pi \cdot 4,05981$ & $2 . \pi \cdot 4,167375$ \\
\hline$\omega_{1}$ & $2 . \pi \cdot 9,15494$ & $2 . \pi \cdot 9,077169$ \\
\hline$\omega_{2}$ & $2 . \pi \cdot 2,27979$ & $2 . \pi \cdot 2,939902$ \\
\hline$\omega_{3}$ & $2 . \pi \cdot 1,22535$ & $2 . \pi \cdot 1,394468$ \\
\hline$\omega_{4}$ & $2 . \pi \cdot 21,9$ & $2 . \pi \cdot 17,31512$ \\
\hline
\end{tabular}

O diagrama de blocos implementado no Simulink ${ }^{\circledR}$ para o filtro de ponderação em frequência é mostrado na figura 13 , a seguir.

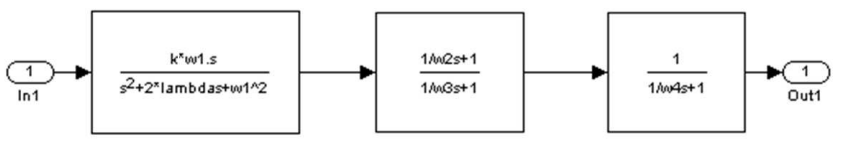

Figura 13: Estrutura do filtro de ponderação em frequência no Simulink ${ }^{\circledR}$.

A resposta em amplitude para o filtro de ponderação em frequência é normalizado para $8,8 \mathrm{~Hz}$, onde se tem a maior sensibilidade de percepção do efeito da cintilação luminosa (ficker) em lâmpadas incandescentes. A figura 14 apresenta a resposta em freqüência do filtro de ponderação em frequência, considerando-se os dois modelos disponíveis de lâmpadas.

Antes de se iniciar um processo de quantificação dos níveis de flicker é extremamente importante se definir o tipo de lâmpada a ser utilizada como referência, de tal forma que os parâmetros do flickermeter possam ser devidamente ajustados. Assim, por exemplo, a utilização do flickermeter modelado para uma lâmpada de referência de $230 \mathrm{~V} / 50 \mathrm{~Hz}$ produziria resultados equivocados em redes elétricas de 120 V/60 Hz.

No caso brasileiro, os sistemas trifásicos possuem apenas tensões fase-neutro padronizadas em 127 Volts ou 220 Volts. Para o caso dos sistemas monofásicos, tem-se ainda tensões fase-neutro em 120 Volts, 115 Volts ou 110 Volts (Prodist, 2010). A grande maioria das cargas de iluminação nacionais, no entanto, estão conectadas a sistemas trifásicos, de tal forma que as tensões fase-neutro disponíveis seriam, de fato, 220 Volts ou 127 Volts. Diante dessa constatação, é importante destacar que a utilização da curva para a lâmpada de referência de $120 \mathrm{~V} / 60 \mathrm{~Hz}$, no caso da rede de 127 $\mathrm{V}$ (fase-neutro), ou da curva da lâmpada de referência de $230 \mathrm{~V} / 50 \mathrm{~Hz}$, no caso da rede de $220 \mathrm{~V}$ (fase-neutro), não produzirá erros representativos no modelo. Em outras palavras, o traçado de uma curva de resposta do filtro de ponderação em frequência (figura 14) para uma lâmpada 


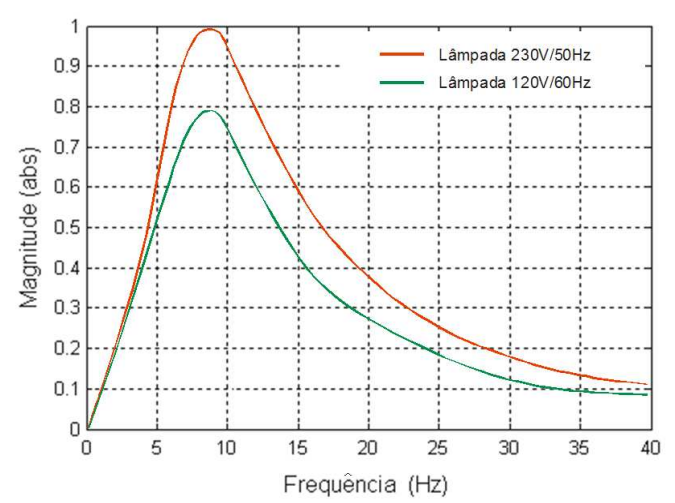

Figura 14: Resposta do filtro de ponderação em frequência normalizado em $8,8 \mathrm{~Hz}$.

de 127 Volts seria praticamente coincidente com a curva obtida para a lâmpada de $120 \mathrm{~V}$. Todos os flickermeters em utilização comercial no Brasil e no mundo possuem apenas curvas para lâmpadas de 120 Volts e 230 Volts.

Finalmente, a curva mostrada na figura 15, ilustra o resultado de saída do bloco 3, segundo a qual é possível observar o período do transitório de acomodação dos filtros digitais.

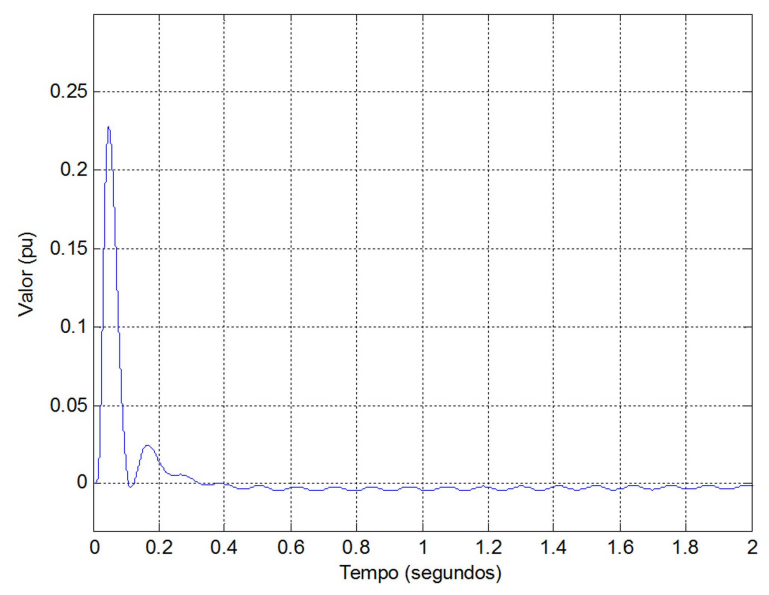

Figura 15: Sinal de saída do bloco 3 (Saída D).

\subsection{Implementação do Bloco 4 (Média quadrática)}

O bloco 4 do protocolo do fickermeter IEC possui duas funções específicas. A primeira delas resume-se na elevação ao quadrado do sinal de saída do bloco 3, simulando a percepção não-linear do comportamento do sistema olho-cérebro frente a variações na iluminação local. A segunda função está relacionada com a simulação do efeito de armazenamento de informações, pelo cérebro humano, relacionadas a variações de iluminação. Em termos práticos, essa função específica é representada por um filtro tipo passa-baixas de $1^{a}$ ordem, com constante de tempo igual a $300 \mathrm{~ms}$ ou, de forma equivalente, com uma frequência de corte igual a $0,5305 \mathrm{~Hz}$. A constante de tempo de $300 \mathrm{~ms}$ representa a característica de acomodação da retina do olho humano às variações de luminosidade.

A função de transferência para esse filtro é indicada conforme a seguir:

$$
F(s)=\frac{1}{0,30 . s+1}
$$

A curva da figura 16 mostra a resposta em frequência para a função de transferência indicada na equação (19).

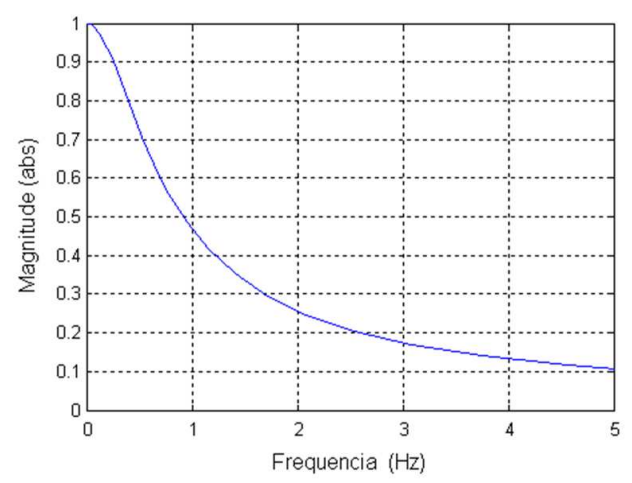

Figura 16: Resposta em frequência do filtro passa-baixas de primeira ordem, com frequência de corte igual a $0,5305 \mathrm{~Hz}$.

O diagrama de blocos resultante da implementação do bloco 4 do fickermeter IEC em ambiente Simulink ${ }^{\circledR}$ é indicado na figura 17, enquanto a a curva mostrada na figura 18 ilustra o resultado de saída do bloco 4 .

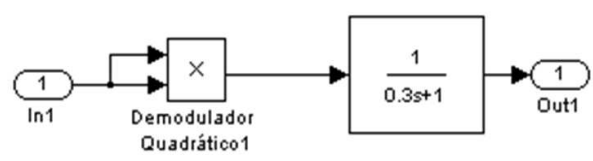

Figura 17: Estrutura do bloco 4 do flickermeter IEC implementado no Simulink ${ }^{\circledR}$.

A sensação instantânea de flicker $(S f)$ é quantificada pelo valor de pico do sinal de saída do bloco 4 (Saída E). Assim, para o caso da figura $18(\mathrm{~b})$, tem-se $S f=1,0 \mathrm{pu}$.

O sinal de saída do bloco 4, em particular, possui um significado importante na metodologia de quantificação de 


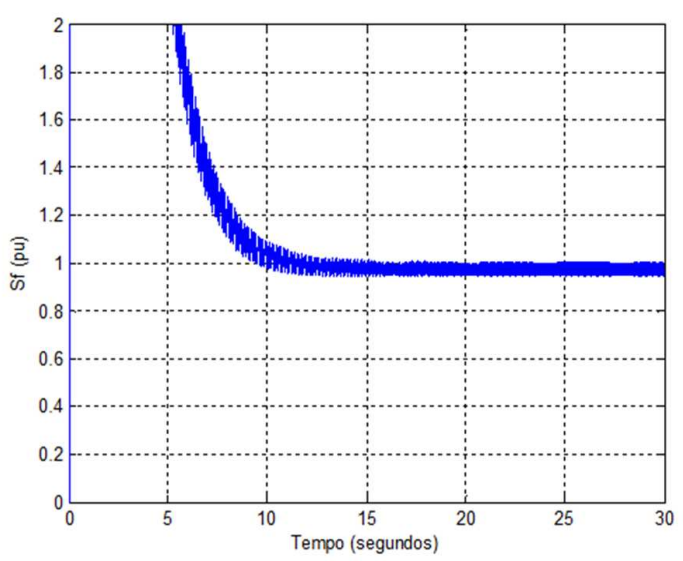

(a)

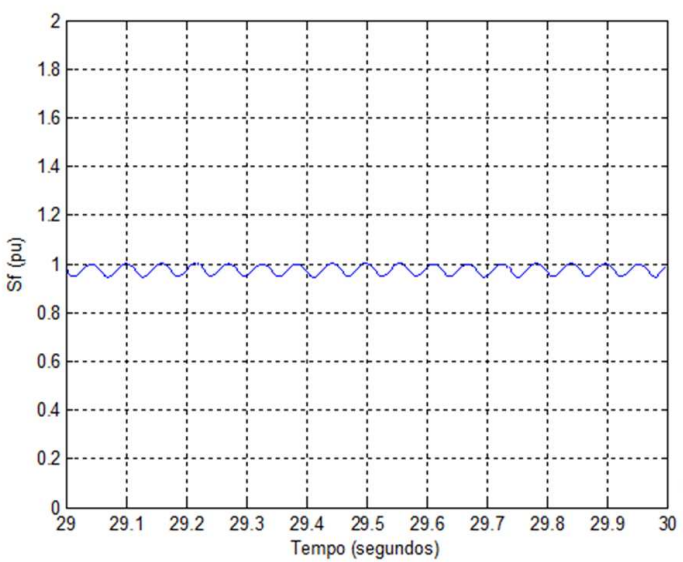

Figura 18: Sinal de saída do bloco 4 (Saída E), (a) 30 segundos de simulação e (b) etapa de regime permanente.

flicker da IEC. Em termos práticos, 1,0 pu de sensação instantânea de flicker (Sf) representa o limiar de sensação de flicker perceptível, a partir do qual 50\% das pessoas acusariam a percepção do fenômeno em uma lâmpada de 60 Watts, $230 \mathrm{~V} / 50 \mathrm{~Hz}$. Conforme pode ser observado na figura 18(a), o tempo total de acomodação de todos os filtros digitais utilizados no modelo do flickermeter é de aproximadamente 15 segundos. Se o tempo de acomodação dos filtros digitais for muito elevado (da ordem de minutos), o flickermeter poderá apresentar resultados insatisfatórios quando de sua aplicação em condições reais de utilização.

\subsection{Implementação do Bloco 5 (Análise estatística)}

A sensação instantânea de flicker ( $S f$ ), representado pelo sinal de saída do bloco 4, assume a forma de um sinal positivo variável, que tende para zero quando a tensão de entrada $(50 / 60 \mathrm{~Hz})$ possui amplitude constante. Devido ao ganho unitário do filtro de ponderação em torno de $8,8 \mathrm{~Hz}$, as flutuações nessa freqüência não são atenuadas e portanto produzem a máxima sensação de flicker. Para as demais frequências as variações de tensão são atenuadas em diferentes graus, dependendo da frequência dos eventos (Deckmann, 1999). Considerando-se que em termos práticos as variações de tensão podem variar tanto em amplitude como em frequência, a sensação instantânea de flicker $(S f)$ varia aleatoriamente, requerendo um tratamento estatístico para sua análise.

Como resultado do tratamento estatístico, é realizada uma medição da severidade de flicker em um período de observação igual a 10 minutos. Em termos práticos, o bloco 5 realiza uma amostragem estatística do sinal de saída do bloco 4 , a cada 10 minutos, organizando as amostras obtidas em classes de acordo com seus valores.

O resultado dessa avaliação estatística é um indicador de probabilidade de curto prazo denominado Pst (Probability Short Term). Matematicamente, esse indicador é definido da seguinte forma:

$$
P s t=\sqrt{0,0314 \cdot P_{0,1}+0,0525 \cdot P_{1 S}+0,0657 \cdot P_{3 S}+0,28 \cdot P_{10 S}+0,08 \cdot P_{50 S}}
$$

Onde:

$P_{i}=$ percentil $i \%$ do sinal amostrado;

Pst $=$ Probability Short Term (Severidade de flicker de curto prazo);

$\mathrm{O}$ sufixo $\mathrm{S}$ nos índices de cada valor percentil indica a necessidade de aplicação de um amortecimento no valor calculado. Apenas para o caso do percentil $\mathrm{P}_{0,1}$, devido ao filtro passa-baixas de $1^{a}$ ordem indicado em (2), não é necessária tal particularidade, uma vez que o mesmo não permite variações bruscas do sinal de entrada para percentil de apenas $0,1 \%$. Para os demais casos, os valores amortecidos são obtidos a partir das seguintes equações:

$$
\begin{gathered}
P_{50 S}=\left(P_{30}+P_{50}+P_{80}\right) / 3 \\
P_{10 S}=\left(P_{6}+P_{8}+P_{10}+P_{13}+P_{17}\right) / 5 \\
P_{3 S}=\left(P_{2,2}+P_{3}+P_{4}\right) / 3 \\
P_{1 S}=\left(P_{0,7}+P_{1}+P_{1,5}\right) / 3
\end{gathered}
$$

Uma vez calculados os indicadores $P s t$, para cada 10 minutos de observação, pode-se também calcular um outro indicador também definido pela IEC, o Probability Long Term (Plt), 
calculado a cada 2 (duas) horas, o qual tem por objetivo promover uma avaliação da severidade de flicker para os casos de várias cargas perturbadoras, geradoras do fenômeno flicker, simultaneamente, e ainda com ciclos de operação de longa duração. Matematicamente, o Plt é calculado conforme abaixo:

$$
P l t=\sqrt[3]{\frac{\sum_{i=1}^{N} P s t_{i}^{3}}{N}}
$$

Onde:

$P s t_{i}=$ valores consecutivos de $P s t(i=1,2,3, \ldots, N)$;

Plt $=$ Probability Long Term .

\subsubsection{Implementação simplificada do bloco 5 do flickermeter IEC}

Para os propósitos do presente trabalho, a implementação do bloco 5 do flickermeter IEC foi realizada de forma simplificada, porém não menos funcional, que a indicada na IEC 61.000-4-15. Aliás, a referida implementação modificada do bloco 5 , pode ser considerada uma contribuição significativa do presente trabalho, reduzindo tempo de processamento e esforço computacional, sem comprometimento da eficácia do modelo, como poderá ser comprovado nos testes de calibração, assim como no desempenho do medidor desenvolvido, apresentados mais adiante.

Basicamente, a implementação do bloco 5 do flickermeter IEC foi realizada no Matlab ${ }^{\circledR}$, através do código indicado no apêndice A, o qual consiste na ordenação do vetor Sf (sensação instantânea de flicker), resultado de saída do bloco 4, seguido do cálculo dos percentis indicados nas equações de (21) a (24). Na realidade os referidos percentis são calculados apenas buscando-se sua posição no vetor ordenado de $S f$.

O algoritmo mostrado na figura 19 ilustra o processo utilizado na modelagem simplificada do bloco 5 . Basicamente, considerando-se que o tamanho do vetor Sf é muito grande (360.000 elementos), cada percentil necessário para os cálculos indicados nas equações (21) a (24) coincidirá com uma posição exata no vetor $S f$ ordenado. Assim, conhecendo-se a referida posição, conhece-se o percentil associado.

Todos os valores de variáveis utilizadas no protocolo do flickermeter IEC, desenvolvido em ambiente Simulink-Matlab ${ }^{\circledR}$ foram concentrados em um única rotina de entrada, conforme mostrado no apêndice $\mathrm{B}$.

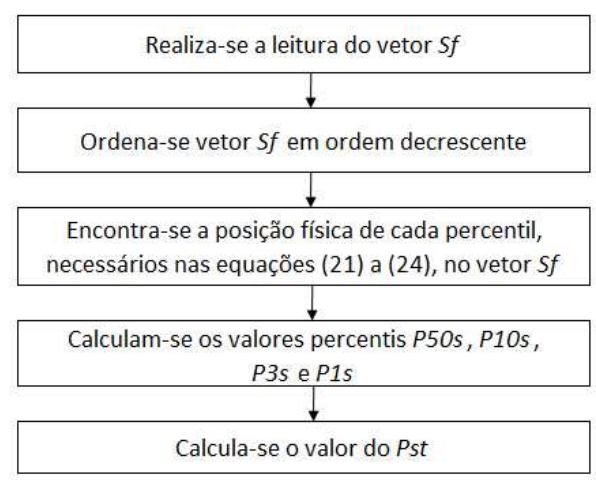

Figura 19: Algoritmo de ordenação do vetor Sf para implementação simplificada do bloco 5 do flickermeter IEC.

\subsubsection{Implementação original do bloco 5 do flickermeter IEC}

O protocolo definido pela IEC 61.000-4-15 estabelece que a implementação do bloco 5, o qual é responsável pelo cálculo do indicador de severidade de flicker de curta duração (Pst), através da análise estatística dos registros de sensação instantânea de flicker ( $S f$ ), deve ser realizada considerando-se uma subdivisão da amplitude dos sinais de sensação instantânea de flicker em um número adequado de classes. Para esse efeito, os níveis de sensação instantânea de ficker $(S f)$ são amostrados a uma taxa constante. Dessa forma, cada vez que um determinado valor limite de classe é alcançado, um contador de tempo específico é incrementado em uma unidade. Ao final desse processo é então obtida a função de probabilidade cumulativa da sensação instantânea de flicker.

Para feito do presente trabalho não serão apresentados os detalhes da implementação do bloco 5 do flickermeter IEC, conforme protocolo original (IEC, 2003). Contudo, a seguir serão apresentados resultados de simulação evidenciando o desempenho das duas formas de implementação consideradas: simplificada e original.

A figura 20, mostra a diferença entre os valores de $P$ st calculados através das duas formas de implementação do bloco 5 do flickermeter IEC.

Conforme pode ser verificado através da figura 20, a implementação do bloco 5 conforme metodologia original definida pela IEC é fortemente dependente do número de classes de estratificação dos valores de sensação instantânea de flicker ( $S f$ ). Por esse motivo, a IEC define que o número de classes a ser utilizado na modelagem do equipamento seja maior ou igual a 64 classes, de forma a minimizar as imprecisões da metodologia. No caso apresentado na figura 20, o qual foi elaborado considerando-se uma frequência de 


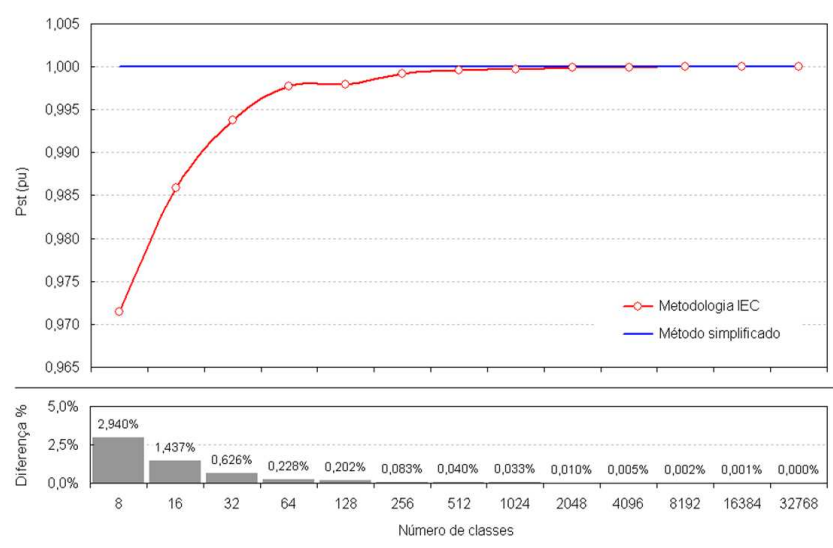

Figura 20: Comparativo de desempenho de diferentes formas de implementação do bloco 5 do flickermeter IEC.

modulação igual a 13,5 Hz e uma amplitude de modulação de 0,547\% (Pst $=1 \mathrm{pu}$ ), o valor do Pst obtido somente se iguala aos valores calculados através da metodologia simplificada para um número de classes muito elevado. Na verdade, pode-se dizer que os valores de Pst são iguais, quando da comparação das duas metodologias, quando o número de classes tende a infinito. A figura 21 apresenta essa mesma análise considerando-se todos os parâmetros sugeridos pela IEC 61.000-4-15 para efeito da realização dos testes de calibração no modelo desenvolvido.

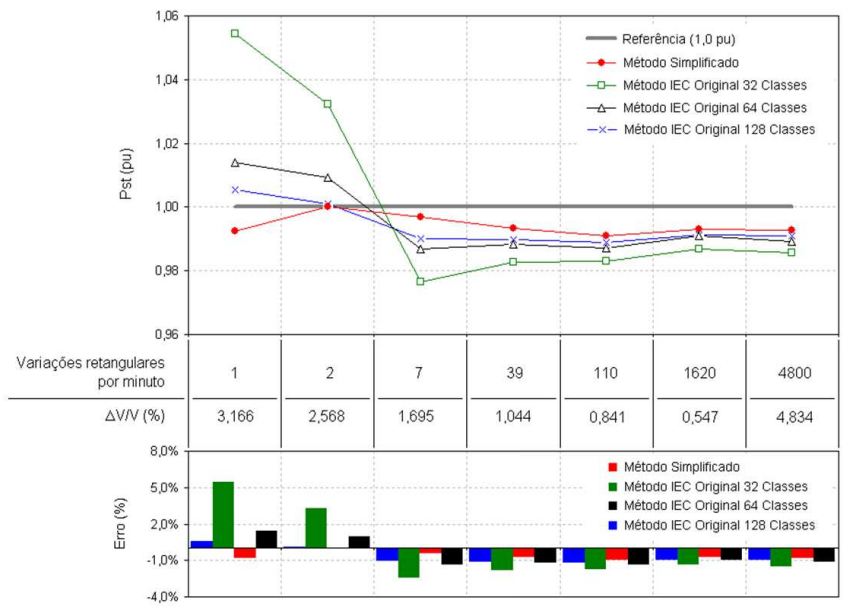

Figura 21: Desempenho das duas formas de implementação do bloco 5 do flickermeter IEC.

Da análise da figura 21, fica mais uma vez evidenciada a grande correlação existente entre o número de classes utilizada na modelagem original do bloco 5 do flickermeter IEC, assim como o excelente desempenho da metodologia simplificada, proposta no presente trabalho. Um aspecto importante refere-se ao esforço computacional necessário para implementação do bloco 5 conforme metodologia IEC, principalmente considerando-se um nível elevado de classes.

Finalmente, a figura 22 apresenta os erros máximos obtidos (sempre comparados à unidade $-P s t=1,0 \mathrm{pu}$ ) quando da implementação do bloco 5 a partir da metodologia simplificada proposta, assim como da metodologia IEC original para três quantidades distintas de classes.

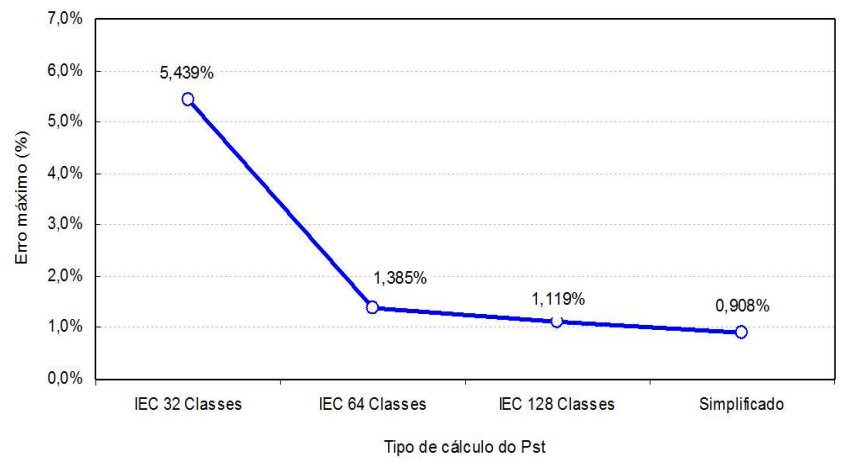

Figura 22: Comparativo dos erros máximos obtidos.

A figura 23 ilustra a redução do tempo de processamento do bloco 5, assim como do esforço computacional, obtida através da implementação simplificada proposta neste trabalho. Os tempos de processamento foram medidos através de comandos específicos (TIC e TOC), destinados para tal propósito, existentes no próprio Matlab ${ }^{\circledR}$. O esforço computacional, por sua vez, foi medido através da utilização do histórico de uso de CPU do Windows ${ }^{\circledR}$, estando todos os demais aplicativos fechados.

No tópico seguinte, serão apresentados os resultados dos testes de calibração do modelo de flickermeter desenvolvido em ambiente Matlab-Simulink ${ }^{\circledR}$, considerando-se, para esse propósito, a implementação simplificada do bloco 5 .

\section{TESTES DE CALIBRAÇÃO NO MODELO DE FLICKERMETER IEC DESENVOLVIDO}

Os testes de calibração no modelo do flickermeter IEC desenvolvido no presente trabalho foram realizados exatamente conforme prescrito na norma IEC 61.000-4-15.

Nesse sentido, foram realizados dois testes específicos. O primeiro considerando apenas o sinal de saída do bloco 4 do flickermeter (Saída E), o qual representa a sensação instantânea de flicker ( $S f$ ), e o segundo considerando-se o valor de Pst (severidade de flicker de curta duração) resultante da saída do bloco 5 (Saída F).

A IEC 61.000-4-15 admite um erro máximo de 5,0\% 

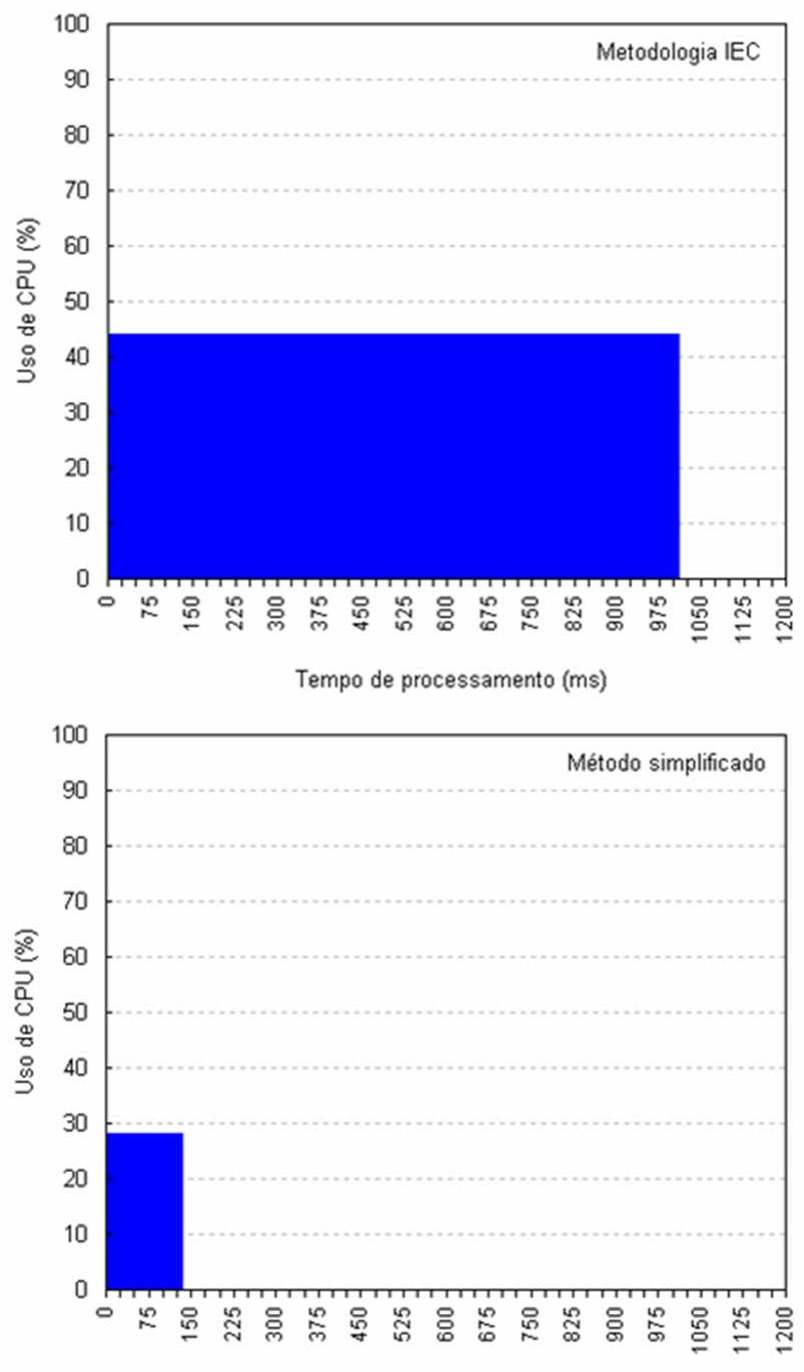

Tempo de processamento (ms)

Figura 23: Análise da redução do tempo de processamento e do esforço computacional resultante da implementação simplificada do bloco 5 .

em relação à unidade $(1,0$ pu) para cada combinação de frequência de modulação e variação de tensão. Nesse caso, a IEC considera flutuações de tensão retangulares para efeito dos referidos testes de calibração.

Para os testes de calibração considerando-se o valor de $S f$ (sensação instantânea de flicker) resultante da saída do bloco 4 (Saída E), o protocolo IEC 61.000-4-15 estabelece uma tabela normalizada $(S f=1,0 \mathrm{pu}$ ) de resposta para flutuações de tensão retangulares, conforme indicado na tabela 2. Para cada caso indicado na referida tabela, o valor de $S f$ deverá estar compreendido entre $1,0 \mathrm{pu} \pm 0,05 \mathrm{pu}$. Ou seja, o erro percentual deverá ser inferior a $\pm 5,0 \%$.
Tabela 2: Parâmetros utilizados para o teste de calibração do modelo do flickermeter IEC - lâmpada $120 \mathrm{~V} / 60 \mathrm{~Hz}$ Sensação instantânea de flicker $(S f)$.

\begin{tabular}{|c|c|}
\hline Frequência $(\mathbf{H z})$ & Variaçäo - $\mathbf{A} \mathbf{V} \mathbf{N} \%$ \\
\hline 0,5 & 0,600 \\
\hline 1,0 & 0,547 \\
\hline 1,5 & 0,504 \\
\hline 2,0 & 0,471 \\
\hline 2,5 & 0,439 \\
\hline 3,0 & 0,421 \\
\hline 3,5 & 0,407 \\
\hline 4,0 & 0,394 \\
\hline 4,5 & 0,371 \\
\hline 5,0 & 0,349 \\
\hline 5,5 & 0,323 \\
\hline 6,0 & 0,302 \\
\hline 6,5 & 0,282 \\
\hline 7,0 & 0,269 \\
\hline 7,5 & 0,258 \\
\hline 8,0 & 0,255 \\
\hline 8,8 & 0,253 \\
\hline 9,5 & 0,257 \\
\hline
\end{tabular}

\begin{tabular}{|c|c|}
\hline Frequência $(\mathbf{H z})$ & Variaçäo $\mathbf{-} \mathbf{A} \mathbf{N}$ \% \\
\hline 10,0 & 0,264 \\
\hline 10,5 & 0,280 \\
\hline 11,0 & 0,297 \\
\hline 11,5 & 0,309 \\
\hline 12,0 & 0,323 \\
\hline 13,0 & 0,369 \\
\hline 14,0 & 0,411 \\
\hline 15,0 & 0,459 \\
\hline 16,0 & 0,513 \\
\hline 17,0 & 0,580 \\
\hline 18,0 & 0,632 \\
\hline 19,0 & 0,692 \\
\hline 20,0 & 0,752 \\
\hline 21,0 & 0,818 \\
\hline 22,0 & 0,853 \\
\hline 23,0 & 0,946 \\
\hline 24,0 & 1,076 \\
\hline 40,0 & 3,460 \\
\hline
\end{tabular}

Após a injeção dos sinais de entrada no bloco 1, de forma a abranger todas as combinações de frequência e amplitude de modulação indicadas na tabela 2 , obteve-se como erro máximo do modelo desenvolvido um valor percentual de $-2,23 \%$, sendo inferior, portanto, ao limite de $5,0 \%$ prescrito pelo protocolo IEC 61.000-4-15. A figura 24 mostra os resultados dos testes de calibração efetuados para a sensação instantânea de flicker $(S f)$.

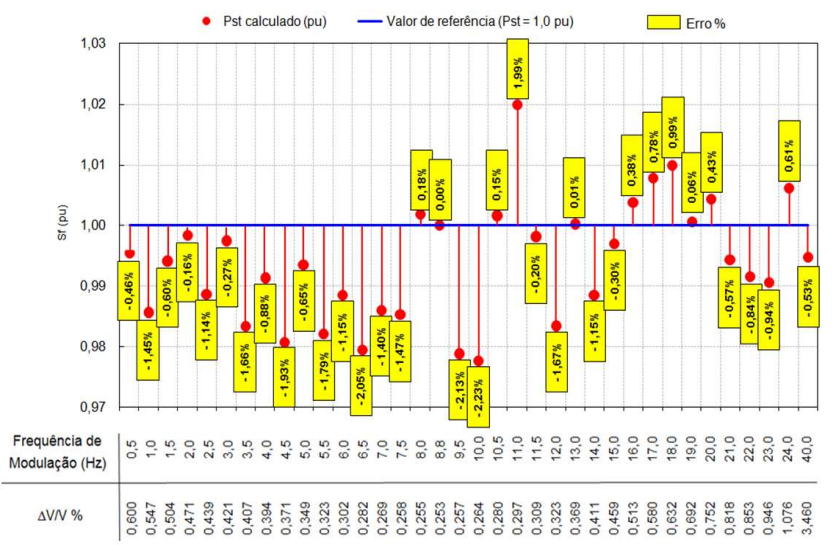

Figura 24: Resultados dos testes de calibração para a sensação instantânea de flicker $(S f)$.

Para os testes de calibração considerando-se o valor de Pst (severidade de flicker de curta duração) resultante da saída do bloco 5 (Saída F), o protocolo IEC 61.000-4-15 estabelece um teste de especificação baseado nos valores de variações retangulares por minuto e variações de tensão indicadas na tabela 3 .

Após a injeção dos sinais de entrada no bloco 1, abrangendo todas as combinações de frequência e amplitude de 
modulação indicadas na tabela 3 , obteve-se como erro máximo do modelo desenvolvido um valor percentual de $-0,908 \%$, muito inferior, portanto, ao limite de $5,0 \%$ prescrito pelo protocolo IEC 61.000-4-15. A figura 25 mostra os resultados dos testes de calibração efetuados para o indicador de severidade de flicker de curta duração $(P s t)$.

Tabela 3: Parâmetros utilizados para o teste de calibração do modelo do flickermeter IEC - lâmpada $120 \mathrm{~V} / 60 \mathrm{~Hz}$ Indicador de severidade de flicker de curta duração (Pst).

\begin{tabular}{|c|c|}
\hline \multicolumn{2}{|c|}{ Lámpada $\mathbf{1 2 0} \mathbf{V} / \mathbf{6 0} \mathbf{H z}$} \\
\hline Variaçöes retangulares por minuto & $\mathbf{\Delta V / N \%}$ \\
\hline 1 & 3,166 \\
\hline 2 & 2,568 \\
\hline 7 & 1,695 \\
\hline 39 & 1,044 \\
\hline 110 & 0,841 \\
\hline 1620 & 0,547 \\
\hline 4000 & - \\
\hline 4800 & 4,834 \\
\hline
\end{tabular}

Com base nos resultados apresentados, portanto, o modelo do flickermeter IEC desenvolvido no presente trabalho se apresenta em conformidade com o protocolo IEC 61.000-4-15, podendo ser utilizado como ferramenta de avaliação da cintilação luminosa (flicker) nos sistemas elétricos, assim como implementado em hardware para concepção de novos flickermeters. $\mathrm{O}$ tópico seguinte apresenta os resultados obtidos decorrentes da implementação em hardware do modelo computacional proposto para o flickermeter IEC.

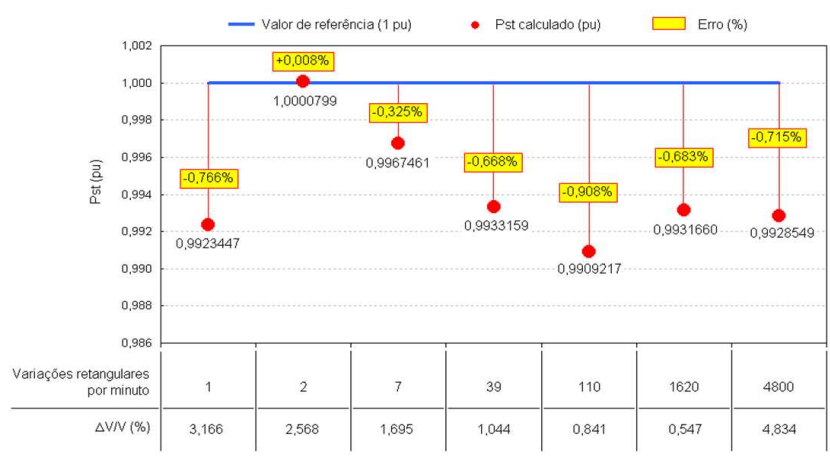

Figura 25: Resultados dos testes de calibração para o indicador Pst (Probability Short Term).

\section{IMPLEMENTAÇÃO EM HARDWARE DO MODELO DE FLICKERMETER DESENVOLVIDO}

Visando uma validação definitiva para o modelo de flickermeter IEC sugerido no presente trabalho, foi desenvolvido, no âmbito de um projeto de Pesquisa e Desenvolvimento junto à Espírito Santo Centrais Elétricas S/A (ESCELSA), um registrador de parâmetros da qualidade da onda da tensão, o qual incorpora o modelo de flickermeter proposto para monitoração do fenômeno da flutuação de tensão. Nesse sentido, os mesmos autores publicaram recentemente (Colnago et al. 2010) um artigo relacionado com o equipamento desenvolvido.

O referido equipamento de monitoração foi concebido para o registro de diversas grandezas elétricas como, distorção harmônica de tensão, variação de tensão em regime permanente, variações de tensão de curta duração, desequilíbrios de tensão, interrupções de fornecimento, além, evidentemente, das flutuações de tensão, objeto da análise proposta.

Para efeito do presente tópico, portanto, serão considerados apenas os aspectos relacionados com as flutuações de tensão. A figura 26 apresenta uma foto do equipamento desenvolvido.

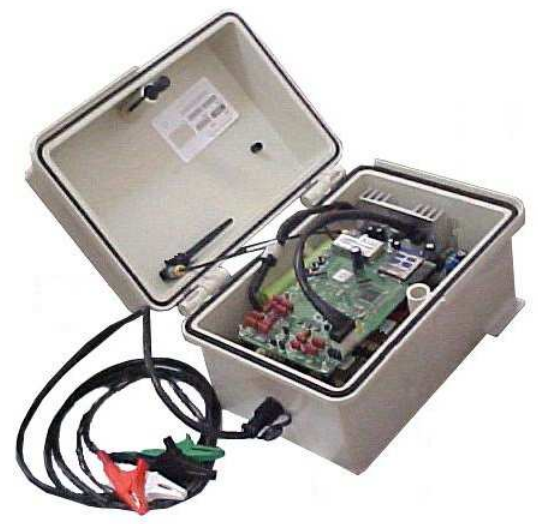

Figura 26: Registrador de parâmetros da qualidade da onda da tensão, contemplando o modelo de flickermeter proposto no presente trabalho.

Para efeito de comparação, o desempenho do medidor (flickermeter) desenvolvido foi comparado com um medidor comercial concebido com características de equipamento Classe A, conforme IEC 61.000-4-30.

Os testes de desempenho operacional para análise da sensação instantânea de flicker (Sf) foram realizados conforme procedimento estabelecidos na IEC 61.000-4-15, através da submissão dos dois medidores às variações de 
tensão e frequência mostradas na tabela 2. Como valor referencial, todos os valores registrados devem retornar uma sensação instantânea de flicker $(S f)$ unitária $(1,0$ pu). Contudo, em função da impossibilidade de utilização de uma fonte devidamente calibrada, segundo a qual seria possível a geração dos níveis de sensação instantânea de flicker adequados para os testes, as comparações seguintes devem consideradas apenas entre os próprios medidores utilizados, não sendo possível uma comparação direta com os parâmetros resultantes da fonte de tensão programável. De qualquer forma, o medidor de referência utilizado nos testes apresenta classificação nível A (conforme IEC 61.000-4-30), o que garante uma excelente referência de comparação para o medidor desenvolvido.

A figura 27 apresenta os resultados do desempenho do medidor desenvolvido, comparativamente ao medidor de referência, para a sensação instantânea de flicker $(S f)$.

Como pode ser observado pela análise da figura 27 , o erro apresentado em relação ao medidor de referência foi de $5,05 \%$, garante uma boa performance para o medidor desenvolvido. Vale ressaltar que mesmo o medidor de referência (Classe A) pode apresentar, segundo a IEC 61.000-4-15, variações de $+/-5,0 \%$ em relação a uma fonte de referência devidamente certificada.
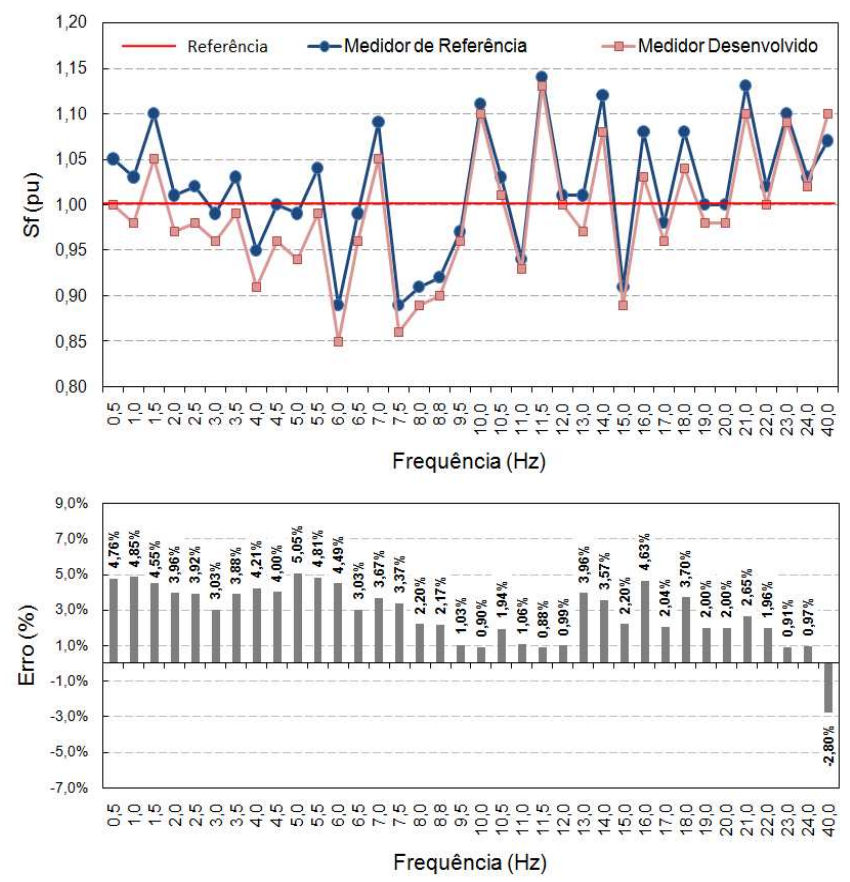

Figura 27: Resultados dos testes comparativos acerca do desempenho do medidor desenvolvido em relação ao indicador de sensação instantânea de flicker (Sf).

Finalmente, a figura 28 apresenta os resultados de desempenho do medidor desenvolvido, comparativamente ao medidor de referência, para o indicador Pst (Probability Short Term).
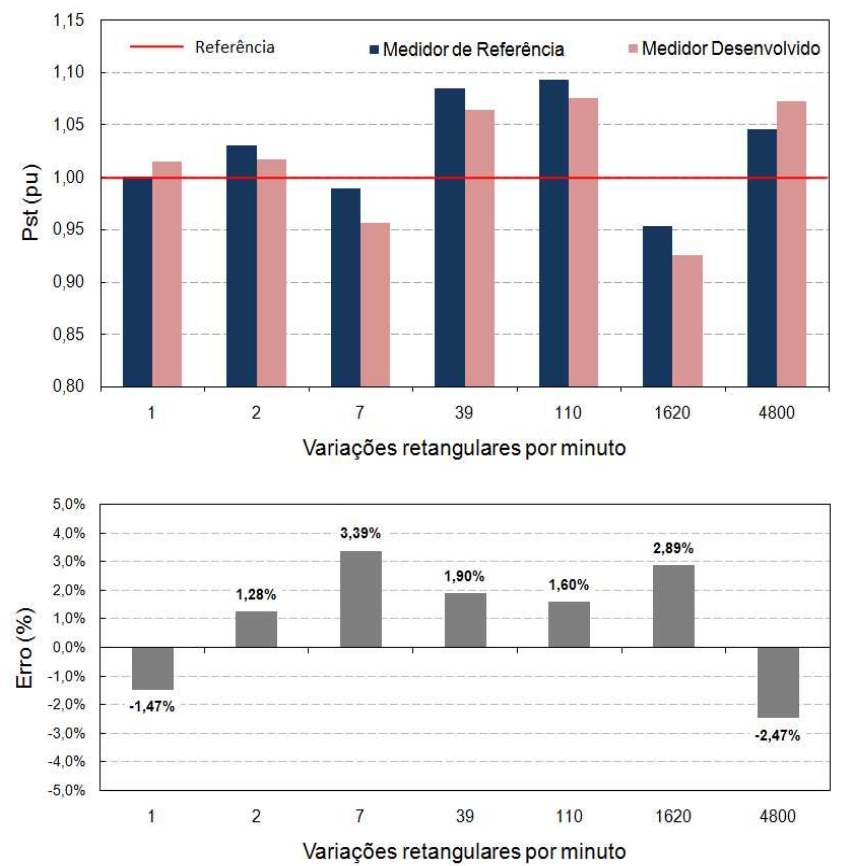

Figura 28: Resultados dos testes comparativos acerca do desempenho do medidor desenvolvido em relação ao indicador Pst (Probability Short Term).

A figura 28 demonstra um excelente desempenho para o medidor desenvolvido, comparativamente ao medidor de referência utilizado, apresentando um erro máximo de $3,39 \%$.

Diante destes resultados, a aplicabilidade do modelo proposto para o flickermeter IEC, conforme apresentado ao longo do presente trabalho, pode ser definitivamente considerada para utilização em trabalhos futuros.

\section{CONCLUSÕES}

O presente trabalho mostrou uma proposta de implementação do protocolo do flickermeter IEC 61.000-4-15 em ambiente Matlab-Simulink ${ }^{\mathbb{R}}$. Em termos práticos, o trabalho representa um tutorial passo-a-passo para implementação do flickermeter IEC. Todas as funções de transferência associadas foram mostradas juntamente com suas respectivas curvas de resposta em frequência. Particularmente para o bloco 5 do flickermeter IEC foi proposta uma implementação simplificada, a qual resultou em uma considerável redução do tempo de processamento, assim como do esforço computacional, comparativamente ao modelo original proposto pela IEC. 
Na sequência foram realizados todos os testes de calibração no modelo, conforme propostos pela IEC. Os resultados mostraram que o modelo apresenta uma performance muito boa, apresentamdo erro máximo de $-0,908 \%$ para a sensação instantânea de flicker $(S f)$ e 2,23\% para o indicador de severidade de flicker de curta duração (Pst).

Finalmente, foi mostrado que o modelo desenvolvido, devidamente implementado em hardware, apresentou um bom desempenho, podendo o mesmo ser utilizado em trabalhos futuros nos quais exista a necessidade de quantificação do fenômeno da flutuação da tensão.

\section{REFERÊNCIAS BIBLIOGRÁFICAS}

Bertola, A.; Lazaroiu, G.C.; Roscia, M.; Zaninelli, D. (2004). A Matlab-Simulink flickermeter model for power quality studies. IEEE PES $11^{\text {th }}$ International Conference on Harmonics and Quality of Power, September 2004, Lake Placid, USA.

Bhim S.; Alka A.; Mittal, A.P.; Gupta J.R.P.(2006), MATLAB-based modelling of distribution STATCOM applied to isolated power system. International Journal of Energy Technology and Policy 2006 - Vol. 4, No.3/4 pp. $274-293$.

Bollen, M. H. J.; Gu, I. Y. H. (2006). Signal Processing of Power Quality Disturbances. IEEE Press Series on Power Engineering, Willey Interscience.

Colnago, G. P. Desenvolvimento e Implementação de um Sistema de Monitoramento em Tempo Real da Tensão da Rede com Acesso Remoto. Dissertação de Mestrado. UFES. Vitória - ES. 2009.

Colnago, G. P.; Vieira, J. L. F; Sousa, G. C. D.; Trabach, E.; Macedo Jr., J. R; Macieira, L. B. B. (2010). Development and implementation of a voltage real-time monitor system of electrical network with remote access. International Conference on Renewable Energies and Power Quality - ICREPQ'10, Granada, Spain, March 2010.

Deckmann, S. M., (1999). Estudos sobre qualidade da energia elétrica. ANEEL. Relatório parcial 2 Flutuações de tensão - Metodologia de avaliação da cintilação.

Deckmann, S.M., Pomilio, J. A. Medição de flicker: Processamento direto e análise RMS. II Seminário Brasileiro sobre Qualidade da Energia Elétrica SBQEE. São Lourenço - MG, 1997.

Deckmann, S. M., Rocco, A. Medição de Cintilação pelo Método UIE. SNPTEE - GSP, Curitiba - PR, 1989.
Freitas, W.; França, André M. (2005). Modelagem e análise dinâmica de dispositivos DSTATCOM usando o SimPowerSystems para Matlab/Simulink. SBA Controle \& Automação, Jun 2005, vol.16, no.2, p.187-199.

IEC International Standard 868. 1986. Flickermeter Functional and Design Specification. First Edition.

IEC International Standard 61.000-4-15, Edition 1.1 (2003). Electromagnetic compatibility (EMC) - Part 4: Testing and measurement techniques - Section 15: Flickermeter - Functional and design specifications.

IEC International Standard 61.000-4-30, Edition 2.0 (2008). Electromagnetic compatibility (EMC) - Part 4-30: Testing and measurement techniques - Power quality measurement methods.

Macedo Jr., José Rubens. Uma à análise das componentes inter-harmônicas e seus efeitos nos indicadores de flutuação de tensão. Tese de Doutorado. UFES. Vitória - ES. 2009.

Macedo Jr., José Rubens; Simonetti, Domingos S. L. Uma contribuição à análise das componentes inter-harmônicas nos sistemas elétricos de potência Parte I. XVII Congresso Brasileiro de Automática CBA 2008, Setembro de 2008, Juiz de Fora, MG.

Macedo Jr., José Rubens; Simonetti, Domingos S. L. Uma contribuição à análise das componentes inter-harmônicas nos sistemas elétricos de potência Parte II. XVII Congresso Brasileiro de Automática CBA 2008, Setembro de 2008, Juiz de Fora, MG.

Macedo Jr., José Rubens; Simonetti, Domingos S. L. As inter-harmônicas e o fenômeno da cintilação luminosa. VIII Conferência Brasileira sobre Qualidade da Energia Elétrica - CBQEE 2009, Agosto de 2009, Blumenau, SC.

Macedo Jr., José Rubens et al. Modelagem computacional do flickermeter IEC em ambiente Matlab-Simulink $\AA$ Parte I. VIII Conferência Brasileira sobre Qualidade da Energia Elétrica - CBQEE 2009, Agosto de 2009, Blumenau, SC;

Macedo Jr., José Rubens et al. Modelagem computacional do flickermeter IEC em ambiente Matlab-Simulink $\mathrm{R}$ Parte II. VIII Conferência Brasileira sobre Qualidade da Energia Elétrica - CBQEE 2009, Agosto de 2009, Blumenau, SC.

Patel, R.; Pagalthivarthi, K. V. (2005). MATLAB-based modelling of power system components in transient stability analysis. International journal of modelling \& simulation, 2005, vol. 25, no1, pp. 43-50. 
Prodist - Procedimentos de Distribuição. Módulo 8 Qualidade da Energia Elétrica. Agência Nacional de Energia Elétrica. 2010.

Rocha, J. P.de Sousa; Deckmann, S.M. Digital Flickermeter Implementation. $38^{\text {th }}$ Midwest Symposium on Circuits and Systems, Brasil , 2, 757-760, (1995).

Ruiz, J., Lazkano, A., Aramendi, E. Leturiondo, L. A. (2000). Analysis of sensitivity to the main parameters involved in the digital implementation of the UIE flickermeter. $10^{\text {th }}$ Mediterranean Electrotechnical Conference, MEleCon 2000, Vol. II.

UIE Report. UIE Flickermeter - Functional and Design Specifications. 1982. International Union for Electro-Heat Disturbances Study Committee - Flicker Measuring Methods Working Group.
APÊNDICE A: Código de programação para implementação simplificada do bloco 5 do flickermeter IEC no Matlab.

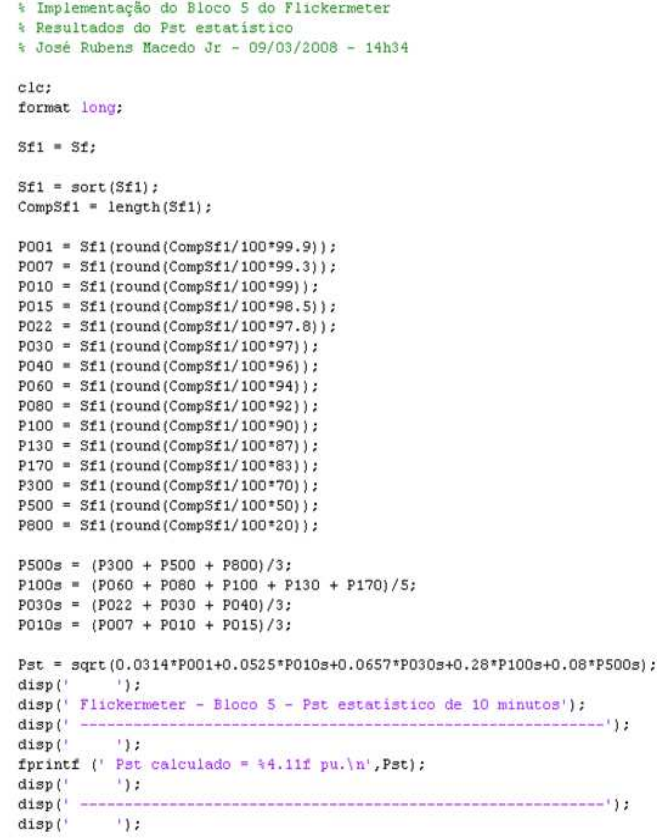

APÊNDICE B: Código de programação para implementação do cartão de entrada do flickermeter IEC no Matlab.

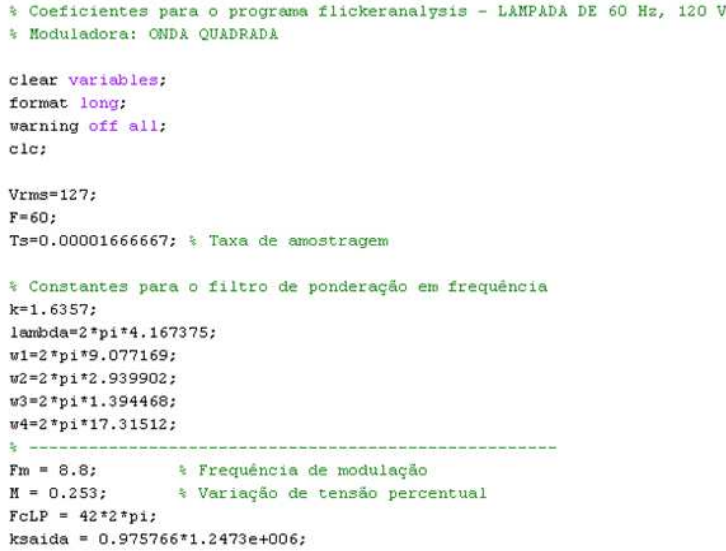

